PROCEEDINGS OF THE

AMERICAN MATHEMATICAL SOCIETY

Volume 132, Number 2, Pages 365-373

S 0002-9939(03)07172-7

Article electronically published on August 28, 2003

\title{
ON ASYMPTOTICALLY NONEXPANSIVE MAPPINGS IN HYPERCONVEX METRIC SPACES
}

\author{
M. A. KHAMSI
}

(Communicated by Jonathan M. Borwein)

\begin{abstract}
Since bounded hyperconvex metric spaces have the fixed point property for nonexpansive mappings, it is natural to extend such a powerful result to asymptotically nonexpansive mappings. Our main result states that the approximate fixed point property holds in this case. The proof is based on the use, for the first time, of the ultrapower of a metric space.
\end{abstract}

\section{INTRODUCTION}

The notion of hyperconvexity is due to Aronszajn and Panitchpakdi [AP] who proved that a hyperconvex space is a nonexpansive absolute retract; i.e., it is a nonexpansive retract of any metric space in which it is isometrically embedded. The corresponding linear theory is well developed and associated with the names of Gleason, Goodner, Kelley and Nachbin (see, for instance, [La]). The nonlinear theory is still developing. The recent interest in these spaces goes back to the results of Sine [Sn1] and Soardi [So] who proved independently that the fixed point property for nonexpansive mappings holds in bounded hyperconvex spaces. Since then many interesting results have been shown to hold in hyperconvex spaces. For more on the metric fixed point property, the interested reader may consult [AK] and [GK] as well as the most recent book [KK].

Recall also that Jawhari, Misane and Pouzet JMP were able to show that Sine and Soardi's fixed point theorem is equivalent to the classical Tarski's fixed point theorem in complete ordered sets. This happens via the notion of generalized metric spaces. Therefore, the notion of hyperconvexity should be understood and appreciated in a more abstract formulation.

In opposition to the lack of linearity, hyperconvexity provides us with a very rich metric structure that leads to a collection of surprising and beautiful results related to different branches of mathematics such as topology, graph theory, multivalued analysis, and fixed point theory.

In this work, we investigate some open questions related to the fixed point property (fpp) in hyperconvex metric spaces. Historically, nonexpansive mappings have enjoyed most of the interest, and were at the core of the fpp in hyperconvex metric spaces. The main motivation for this work was a question by Kirk [Ki2] of whether

Received by the editors March 12, 2002.

2000 Mathematics Subject Classification. Primary 47H09, 47H10.

Key words and phrases. Nonexpansive mappings, asymptotically nonexpansive mappings, fixed point, hyperconvex. 
asymptotically nonexpansive mappings have the fpp in bounded hyperconvex metric spaces. This question is still open. But we were able to show that the asymptotic fixed point property holds in this case. The proof is nonstandard in nature and uses the notion of ultrapower of a metric space. To the best of our knowledge, this is the first time that such a notion is considered in the metric setting, which leads to some positive new results.

\section{BASIC DEFINITIONS}

A metric space $M$ is said to be hyperconvex if given any family $\left\{x_{\alpha}\right\}$ of points of $M$ and any family $\left\{r_{\alpha}\right\}$ of real numbers satisfying

$$
d\left(x_{\alpha}, x_{\beta}\right) \leq r_{\alpha}+r_{\beta},
$$

it is the case that $\bigcap_{\alpha} B\left(x_{\alpha} ; r_{\alpha}\right) \neq \emptyset$.

The fundamental result of $\mathrm{AP}$ asserts that a metric space $M$ is hyperconvex if and only if it is injective. Thus $M$ is hyperconvex if given any two metric spaces $X$ and $Y$ with $Y$ a subspace of $X$, and any nonexpansive mapping $f: Y \rightarrow M$, then $f$ has a nonexpansive extension $\tilde{f}: X \rightarrow M$. Basic results about injective metric spaces can be found in [IS.

An admissible subset of $M$ is a set of the form

$$
\bigcap_{i} B\left(x_{i} ; r_{i}\right)
$$

where $\left\{B\left(x_{i} ; r_{i}\right)\right\}$ is a family of closed balls centered at points $x_{i} \in M$ with respective radii $r_{i}$. It is quite easy to see that an admissible subset of a hyperconvex metric space is hyperconvex. In what follows we use $\mathcal{A}(M)$ to denote the family of all nonempty admissible subsets of $M$.

The recent interest into hyperconvexity goes back to the results of Sine $\mathrm{Sn1}$ and Soardi $[\mathrm{So}]$ who proved that if $H$ is a bounded hyperconvex metric space and $T: H \rightarrow H$ is nonexpansive, i.e., $d(T(x), T(y)) \leq d(x, y)$ for any $x, y \in H$, then there exists a fixed point $x \in H$, i.e., $T(x)=x$. Moreover, the fixed point set $\operatorname{Fix}(T)$ is hyperconvex and, consequently, is a nonexpansive retract of $H$.

Perhaps the most elegant result in this direction belongs to Baillon [Ba], who proved that the conclusion of the results of Sine and Soardi is still valid when dealing with any family of commutative nonexpansive mappings. In fact, his proof is based on the following structural result:

Theorem. Let $H$ be a bounded hyperconvex metric space. If $\left\{H_{i}\right\}_{i \in I}$ is a decreasing family of nonempty hyperconvex subsets of $H$, then we have

$$
\bigcap_{i \in I} H_{i} \neq \emptyset
$$

and it is hyperconvex.

The proof is nonintuitive and very complicated.

When $H$ is not bounded, then a nonexpansive mapping may not have a fixed point. But it is not hard to see that the nonexpansive mapping $T$ always has an approximate fixed point, i.e.,

$$
\inf \{d(x, T(x)) ; x \in H\}=0 .
$$


When a map satisfies the above, we say that $T$ satisfies the approximate fixed point property. In this case, the set

$$
H_{\varepsilon}=\{x \in H ; d(x, T(x)) \leq \varepsilon\}
$$

is not empty for any $\varepsilon>0$. In fact, Sine $\underline{\operatorname{Sn} 2}$ proved that $H_{\varepsilon}$ is hyperconvex.

Next, we discuss convexity in hyperconvex metric spaces. Historically there are two approaches to this. One is based on Penot's ideas $[\mathrm{Pe}$, which were based on the notion of convexity structures and which gave the first interesting generalization of the classical Kirk's fixed point theorem [Ki1] in metric spaces. The other one mimics linear convexity. Here we will use that one. In order to better understand it, we will use a natural embedding of any metric space $M$ in the Banach space $l_{\infty}(M)$ (see EK for more on this). So, if $H$ is hyperconvex, then there exists a nonexpansive retract $R: l_{\infty}(H) \rightarrow H$. For any $x, y \in H$, we write

$$
t x \oplus(1-t) y=R(t x+(1-t) y)
$$

for any $t \in[0,1]$. Here we are using the linear convexity of $l_{\infty}(H)$. It is not hard to check that for any $z \in H$ we have

$$
d(z, t x \oplus(1-t) y) \leq t d(z, x)+(1-t) d(z, y)
$$

for any $t \in[0,1]$.

\section{ULTRAPOWER OF METRIC SPACES}

Let $(M, d)$ be a bounded metric space and $\mathcal{U}$ a nontrivial ultrafilter on the natural numbers. Consider the cartesian product $\mathcal{M}=\prod_{n \geq 1} M$. Define the equivalence relation $\sim$ on $\mathcal{M}$ by

$$
\left(x_{n}\right) \sim\left(y_{n}\right) \text { if and only if } \lim _{\mathcal{U}} d\left(x_{n}, y_{n}\right)=0 .
$$

The limit over $\mathcal{U}$ exists since $M$ is bounded. Then we consider the quotient set $\widetilde{M}$. An element $\tilde{x} \in \widetilde{M}$ is a subset of $\mathcal{M}$. If $\left(x_{n}\right) \in \tilde{x}$, then $\left(y_{n}\right) \in \tilde{x}$ if and only if $\lim _{\mathcal{U}} d\left(x_{n}, y_{n}\right)=0$. On $\widetilde{M}$ define the metric $\tilde{d}$ by

$$
\tilde{d}(\tilde{x}, \tilde{y})=\lim _{\mathcal{U}} d\left(x_{n}, y_{n}\right)
$$

where $\left(x_{n}\right)$ (resp. $\left.\left(y_{n}\right)\right)$ is any element in $\tilde{x}$ (resp. $\left.\tilde{y}\right)$. It is easy to see that $\tilde{d}$ defines a metric on $\widetilde{M}$ which has many nice properties similar to the linear ultrapower of a Banach space. For example, it is obvious that $M$ is isometric to a subset of $\widetilde{M}$. Indeed, let

$$
\dot{M}=\left\{\widetilde{\left(x_{n}\right)} ; x_{n}=x \text { for any } n \geq 1\right\} .
$$

Then it is easy to show that $M$ and $\dot{M}$ are isometric. In the sequel we will use the notation $M=\dot{M}$ and see $x \in M$ as an element of $\widetilde{M}$ as well. Also, it is worth mentioning that if $M$ is complete, then $\widetilde{M}$ is complete. The proof is similar to the linear one. In the linear case it is known that if $X$ is a finite-dimensional Banach space, then $\widetilde{X}$ is also a finite-dimensional Banach space with the same dimension as $X$. The analogue of this is the following result.

Proposition 1. If $M$ is compact, then $\widetilde{M}$ is also compact and isometric to $M$. 
Proof. Since $M$ is compact, then for any sequence $\left(x_{n}\right) \in M$ the $\operatorname{limit} \lim _{\mathcal{U}} x_{n}=x$ exists (in $M$ ) since $\mathcal{U}$ is an ultrafilter. So we have

$$
\lim _{\mathcal{U}} d\left(x_{n}, x\right)=0
$$

or, equivalently, $\widetilde{\left(x_{n}\right)}=x$. Hence $\widetilde{M}$ is a subset of $\dot{M}$, i.e., $\widetilde{M}=\dot{M}$. Therefore, $\widetilde{M}$ is isometric to $M$ and must be compact.

Clearly, one may then ask, what if $M$ is not compact. In this case it is natural to use measures of noncompactness. The most commonly used were introduced by Hausdorff and Kuratowski (see ADL for more details).

Definition 1. Let $(M, d)$ be a metric space and let $\mathcal{B}(M)$ denote the collection of nonempty, bounded subsets of $M$.

(1) The Kuratowski measure of noncompactness $\alpha: \mathcal{B}(M) \rightarrow[0, \infty)$ is defined by

$$
\alpha(A)=\inf \left\{\varepsilon>0 ; A \subset \bigcup_{i=1}^{i=n} A_{i} \text { with } A_{i} \in \mathcal{B}(M) \text { and } \operatorname{diam}\left(A_{i}\right) \leq \varepsilon\right\} .
$$

(2) The Hausdorff (or ball) measure of noncompactness $\chi: \mathcal{B}(M) \rightarrow[0, \infty$ ) is defined by

$$
\chi(A)=\inf \left\{r>0 ; A \subset \bigcup_{i=1}^{i=N} B\left(x_{i}, r\right) \text { with } x_{i} \in M\right\},
$$

where $B(x, r)$ denotes the closed ball centered at $x$ with radius $r$.

We have the following more general result:

Proposition 2. Let $A$ be a bounded subset of $M$. Set $\widetilde{A}=\left\{\widetilde{\left(x_{n}\right)} ; x_{n} \in A\right\}$. Then we have

$$
\chi(A)=\chi(\widetilde{A}) .
$$

Proof. Let $\varepsilon>\chi(A)$ and $\delta>0$. Then by definition of $\chi$, there exists a finite set $D=\left\{x_{1}, x_{2}, \cdots, x_{N}\right\}$ such that

$$
A \subset \bigcup_{i=1}^{i=N} B\left(x_{i}, \varepsilon\right) .
$$

Consider $\widetilde{D}$. Our previous result implies that $\widetilde{D}$ is compact. So there exists a finite set $\left\{\tilde{x}_{1}, \tilde{x}_{2}, \cdots, \tilde{x}_{K}\right\}$ such that

$$
\widetilde{D} \subset \bigcup_{i=1}^{i=K} B\left(\tilde{x}_{i}, \delta\right) .
$$

From here it is easy to see that

$$
\widetilde{A} \subset \bigcup_{i=1}^{i=K} B\left(\tilde{x}_{i}, \varepsilon+\delta\right),
$$

which implies

$$
\chi(\widetilde{A}) \leq \varepsilon+\delta .
$$


Since $\delta$ was chosen arbitrarily, then we have $\chi(\widetilde{A}) \leq \varepsilon$, which implies

$$
\chi(\widetilde{A}) \leq \chi(A) \text {. }
$$

In order to complete our proof, we need to show that $\chi(A) \leq \chi(\widetilde{A})$. Let $\varepsilon>0$. Set $r=\chi(\widetilde{A})+\varepsilon$. Then there exist $\tilde{x}_{i}(i=1, \cdots, K)$ in $\tilde{A}$ such that

$$
\widetilde{A} \subset \bigcup_{i=1}^{i=K} B\left(\tilde{x}_{i}, r\right) .
$$

Set $\left.\tilde{x}_{i}=\widetilde{\left(x_{i}(n)\right.}\right)$, for $i=1, \cdots, K$, with $x_{i}(n) \in A$. We claim that for any $\delta>0$ there exists $n_{0} \geq 1$ such that

$$
A \subset \bigcup_{i=1}^{i=K} B\left(x_{i}\left(n_{0}\right), r+\delta\right) .
$$

Assume not. Then there exists $\delta_{0}>0$ such that for any $n \geq 1$, there exists $x_{n} \in A$ that satisfies

$$
x_{n} \notin \bigcup_{i=1}^{i=K} B\left(x_{i}(n), r+\delta_{0}\right) \text {. }
$$

Set $\tilde{x}=\widetilde{(x(n))} \in \tilde{A}$. Then

$$
\tilde{d}\left(\tilde{x}, \tilde{x}_{i}\right)=\lim _{\mathcal{U}} d\left(x_{n}, x_{i}(n)\right) \geq r+\delta_{0}
$$

for $i=1, \cdots, K$. Clearly, we have

$$
\tilde{x} \notin \bigcup_{i=1}^{i=K} B\left(\tilde{x}_{i}, r\right),
$$

which is our desired contradiction. So letting $\delta>0$, we know that there exists $n_{0} \geq 1$ such that

$$
A \subset \bigcup_{i=1}^{i=K} B\left(x_{i}\left(n_{0}\right), r+\delta\right)
$$

This clearly implies

$$
\chi(A) \leq r+\delta=\chi(\widetilde{A})+\varepsilon+\delta
$$

Since $\epsilon$ and $\delta$ were chosen arbitrarily positive, we conclude that

$$
\chi(A) \leq \chi(\widetilde{A})
$$

which completes the proof of our proposition.

When $M$ is not compact, more can be said about the ultrapower.

Proposition 3. Assume $M$ is not compact. Then $\widetilde{M}$ is not separable.

Proof. Assume $M$ is not compact. Then there exists a bounded sequence $\left(x_{n}\right)$ with no convergent subsequence. In particular, $\lim _{\mathcal{U}} x_{\phi(n)}$ does not exist for any subsequence $\left(x_{\phi(n)}\right)$ of $\left(x_{n}\right)$. Moreover, we can assume that there exists $\varepsilon>0$ such that

$$
\operatorname{sep}\left(x_{n}\right)=\inf \left\{d\left(x_{n}, x_{m}\right) ; n \neq m\right\} \geq \varepsilon .
$$

For any subsequence $\left(x_{\phi(n)}\right)$ of $\left(x_{n}\right)$ set $\tilde{x}_{\phi}=\widetilde{\left(x_{\phi(n)}\right)}$. Clearly, we have

$$
\tilde{d}\left(\tilde{x}_{\phi}, \tilde{x}_{\alpha}\right)=\lim _{\mathcal{U}} \tilde{d}\left(x_{\phi(n)}, x_{\alpha(n)}\right) \geq \varepsilon .
$$


Since any sequence has uncountably many subsequences, the above result implies that $\widetilde{M}$ has an uncountable $\varepsilon$-separated set. Therefore, $\widetilde{M}$ is not separable.

It is quite an amazing result since a linear version of it is also known.

Next we discuss how Lipschitzian mappings extend naturally to the ultrapower. Indeed, let $T: M \rightarrow M$ be a Lipschitzian mapping with $L$ as a constant of Lipschitz, i.e.,

$$
d(T(x), T(y)) \leq L d(x, y) \text { for } x, y \in M .
$$

Then

$$
\lim _{\mathcal{U}} d\left(x_{n}, y_{n}\right)=0 \text { implies } \lim _{\mathcal{U}} d\left(T\left(x_{n}\right), T\left(y_{n}\right)\right)=0 .
$$

This obviously implies that $\tilde{T}: \widetilde{M} \rightarrow \widetilde{M}$ defined by

$$
\tilde{T}\left(\widetilde{\left(x_{n}\right)}\right)=\left(\widetilde{T\left(x_{n}\right)}\right)
$$

is well defined. It is easy to check that $\tilde{T}$ is Lipschitzian with $L$ as a constant of Lipschitz. We also have

$$
\tilde{T}(x)=T(x) \text { for any } x \in M .
$$

Before we jump to the next section where the main result of this work will be stated, it is worth mentioning that hyperconvexity is not a super-property; i.e., the ultrapower of a hyperconvex metric space is not necessarily hyperconvex.

For more on ultrapowers and nonstandard techniques, the interested reader is advised to consult $[\mathrm{AK}]$ and $[\mathrm{Sm}$.

\section{MAIN RESULT}

Before we state the main result of this work, we will need some definitions. Let $M$ be a metric space. A map $T: M \rightarrow M$ is said to be asymptotically nonexpansive if there exists a sequence of positive numbers $\left\{k_{n}\right\}$, with $\lim _{n \rightarrow \infty} k_{n}=1$, such that

$$
d\left(T^{n}(x), T^{n}(y)\right) \leq k_{n} d(x, y) \text { for any } x, y \in M \text { and } n=1,2, \cdots .
$$

The main result of our work goes as follows:

Theorem. Let $H$ be a bounded hyperconvex metric space and $T: H \rightarrow H$ an asymptotically nonexpansive mapping. Then $T$ has approximate fixed points, i.e.,

$$
\inf \{d(x, T(x)) ; x \in H\}=0 \text {. }
$$

Proof. In order to prove the above conclusion, we need to show that for any $\varepsilon>0$, there exists $x \in H$ such that

$$
d(x, T(x)) \leq \varepsilon .
$$

Using the metric convexity of $H$, we define the map

$$
T_{n}=\frac{1}{k_{n}} T^{n} \oplus\left(1-\frac{1}{k_{n}}\right) x_{0}
$$

where $x_{0}$ is a fixed point in $H$ and $k_{n}$ is the Lipschitz constant of $T^{n}$. The maps $\left\{T_{n}\right\}$ are nonexpansive. Consider the ultrapower $\widetilde{H}$ of $H$, over a nontrivial ultrafilter $\mathcal{U}$. Define the operators $\hat{T}$ and $\tilde{T}$ by

$$
\hat{T}(\tilde{x})=\hat{T}\left(\widetilde{\left(x_{n}\right)}\right)=\left(\widetilde{T^{n}\left(x_{n}\right)}\right) \text { and } \tilde{T}(\tilde{x})=\tilde{T}\left(\widetilde{\left(x_{n}\right)}\right)=\left(\widetilde{T\left(x_{n}\right)}\right) .
$$


Since $T$ is an asymptotically nonexpansive mapping, the map $\hat{T}$ is nonexpansive. Moreover, we have

$$
\hat{T}\left(\widetilde{\left(x_{n}\right)}\right)=\left(\widetilde{T_{n}\left(x_{n}\right)}\right) .
$$

Since $T_{n}$ is nonexpansive, Sine and Soardi's fixed point theorem implies the existence of a fixed point $x_{n}$ (of $T_{n}$ ). The point $\tilde{x}=\left(x_{n}\right)$ is a fixed point of $\hat{T}$. Hence the fixed point set $\operatorname{Fix}(\hat{T})$ is a nonempty subset of $\widetilde{H}$. Since the two operators $\hat{T}$ and $\tilde{T}$ commute, then $\tilde{T}$ leaves invariant the set $F i x(\hat{T})$. It is easy to show that $\tilde{T}$ restricted to Fix $(\hat{T})$ is in fact an isometry (in particular, it is nonexpansive). Fix $\varepsilon>0$. Let $\tilde{x}_{i} \in \operatorname{Fix}(\hat{T}), i=1, . ., N$. If $\left.\tilde{x}_{i}=\widetilde{\left(x_{n}(i)\right.}\right)$, for $i=1, \cdots, N$, set

$$
\varepsilon_{n}=\max _{1 \leq i \leq N} d\left(x_{n}(i), T_{n}\left(x_{n}(i)\right)\right) .
$$

Then we have

$$
\lim _{\mathcal{U}} \varepsilon_{n}=0
$$

Set

$$
H_{n}=\left\{x \in H ; d\left(x, T_{n}(x)\right) \leq \varepsilon_{n}\right\} .
$$

Then $H_{n} \neq \emptyset$ because $x_{n}(i) \in H_{n}$, for $i=1, . ., N$. Since $T_{n}$ is nonexpansive, Sine Sn2] proved that $H_{n}$ is hyperconvex. Therefore, there exists

$$
z_{n}(i)=\varepsilon x_{n}(1) \oplus(1-\varepsilon) x_{n}(i) \in H_{n}
$$

for $i=1, . ., N$. Consider, the point

$$
\tilde{z}_{i}=\widetilde{\left(z_{n}(i)\right)}
$$

which we will denote $\varepsilon \tilde{x}_{1} \oplus(1-\varepsilon) \tilde{x}_{i}$. Then we have $\tilde{z}_{i} \in F i x(\hat{T})$, and

$$
d\left(\tilde{z}_{i}, \tilde{z}_{j}\right) \leq(1-\varepsilon) d\left(\tilde{x}_{i}, \tilde{x}_{j}\right)
$$

for $i, j=2, . ., N$. Back to our maps $\hat{T}$ and $\tilde{T}$. Let $\tilde{x} \in F i x(\hat{T})$. Write $\tilde{x}=\tilde{x}_{1}$. Then from the above ideas, there exists $\tilde{x}_{2} \in F i x(\hat{T})$ such that

$$
\tilde{x}_{2}=\varepsilon \tilde{x}_{1} \oplus(1-\varepsilon) \tilde{T}\left(\tilde{x}_{1}\right) .
$$

By induction, we will construct a sequence $\left(\tilde{x}_{n}\right)$ of points in Fix $(\hat{T})$ defined by

$$
\tilde{x}_{n+1}=\varepsilon \tilde{x}_{1} \oplus(1-\varepsilon) \tilde{T}\left(\tilde{x}_{n}\right) .
$$

We have for any $n<m$,

$$
d\left(\tilde{x}_{n}, \tilde{x}_{m}\right) \leq(1-\varepsilon) d\left(\tilde{T}\left(\tilde{x}_{n-1}\right), \tilde{T}\left(\tilde{x}_{m-1}\right)\right)
$$

and, since $\tilde{T}$ is nonexpansive when restricted to $\operatorname{Fix}(\hat{T})$, we get

$$
d\left(\tilde{x}_{n}, \tilde{x}_{m}\right) \leq(1-\varepsilon) d\left(\tilde{x}_{n-1}, \tilde{x}_{m-1}\right) .
$$

This clearly implies that the sequence $\left(\tilde{x}_{n}\right)$ is a Cauchy sequence. Hence it converges to $\tilde{\omega} \in \operatorname{Fix}(\hat{T})$. Moreover, we have

$$
d(\tilde{\omega}, \tilde{T}(\tilde{\omega}))=\lim _{n \rightarrow \infty} d\left(\tilde{x}_{n+1}, \tilde{T}\left(\tilde{x}_{n}\right)\right) \leq \varepsilon \lim _{n \rightarrow \infty} d\left(\tilde{x}_{1}, \tilde{T}\left(\tilde{x}_{n}\right)\right) .
$$


If we set $\delta=\operatorname{diameter}(H)$, we get

$$
d(\tilde{\omega}, \tilde{T}(\tilde{\omega})) \leq \varepsilon \delta .
$$

Therefore, we have proved that for any $\varepsilon>0$, there exists $\tilde{\omega}_{\varepsilon} \in$ Fix $(\hat{T})$, such that

$$
d\left(\tilde{\omega}_{\varepsilon}, \tilde{T}\left(\tilde{\omega}_{\varepsilon}\right)\right) \leq \varepsilon
$$

From this it is easy to extract $x_{\varepsilon} \in H$ such that

$$
d\left(x_{\varepsilon}, T\left(x_{\varepsilon}\right)\right) \leq \varepsilon
$$

for any $\varepsilon>0$.

Remark. Recall that Kirk's original question was about the existence of a fixed point for such mappings. This problem is still open. But one may use a simple embedding of any metric space $M$ into $l_{\infty}(M)$ (see EK for more on this), to show that, in fact, the main problem described here is equivalent to the same problem for the unit ball of $l_{\infty}$. It is worth mentioning that the existence of a fixed point for asymptotically nonexpansive mappings is closely related to the existence of a fixed point for $k$-uniformly Lipschitzian mappings in the linear case (see $[\mathrm{KX}]$ for more on this).

The author wishes to thank the referee for valuable comments on the final version of this work.

\section{REFERENCES}

[AK] A. G. Aksoy and M. A. Khamsi, Nonstandard Methods in Fixed Point Theory, SpringerVerlag, New York, Berlin (1990). MR 91i:47073

[AP] N. Aronszajn and P. Panitchpakdi, Extension of uniformly continuous transformations and hyperconvex metric spaces, Pacific J. Math. 6 (1956), 405-439. MR 18:917c

[ADL] J. M. Ayerbe Toledano, T. Dominguez Benavides, and G. Lopez Acedo, Measures of Noncompactness in Metric Fixed Point Theory, Operator Theory: Advances and Applications 99, Birkhäuser-Verlag, Basel, 1997. MR 99e:47070

[Ba] J. B. Baillon, Nonexpansive mapping and hyperconvex spaces, Contemp. Math. 72 (1988), 11-19. MR 89k:54068

[EK] R. Espinola and M. A. Khamsi, Introduction to hyperconvex spaces, Handbook of Metric Fixed Point Theory, W. A. Kirk and B. Sims, Editors, Kluwer Academic Publishers, Dordrecht, 2001. MR 2003g:47099

[GK] K. Goebel and W. A. Kirk, Topics in Metric Fixed Point Theory, Cambridge Univ. Press, Cambridge (1990). MR 92c:47070

[Is] J. R. Isbell, Six theorems about injective metric spaces, Comment. Math. Helvetici 39 (1964), 65-76. MR 32:431

[JMP] E. Jawhari, D. Misane and M. Pouzet, Retracts: graphs and ordered sets from the metric point of view, Contemp. Math. 57 (1986), 175-226. MR 88i:54022

[KK] M. A. Khamsi, and W. A. Kirk, An Introduction to Metric Spaces and Fixed Point Theory, Pure and Applied Mathematics, Wiley Interscience, New York (2001). MR 2002b:46002

[KX] T. H. Kim and H. K. Xu, Remarks on asymptotically nonexpansive mappings, Nonlinear Analysis 41 (2000), 405-415. MR 2001b:47089

[Ki1] W. A. Kirk, A fixed point theorem for mappings which do not increase distances, Amer. Math. Monthly 72 (1965), 1004-1006. MR 32:6436

[Ki2] W. A. Kirk, Personal Communication.

[La] H. E. Lacey, The Isometric Theory of Classical Banach Spaces, Die Grundlehren der mathematischen Wissenschaften, Band 208, Springer-Verlag, Berlin, Heidelberg, New York (1974). MR 58:12308

[Pe] J. P. Penot, Fixed point theorems without convexity, Bull. Soc. Math. France Mémoire 60 (1979), 129-152. MR 81c:47061 
[Sm] B. Sims, "Ultra"-techniques in Banach Space Theory, Queen's Papers in Pure and Appl. Math., 60, Queen's University, Kingston, Ontario (1982). MR 86h:46032

[Sn1] R. C. Sine, On linear contraction semigroups in sup norm spaces, Nonlinear Anal. 3 (1979), 885-890. MR 80i:47082

[Sn2] R. C. Sine, Hyperconvexity and approximate fixed points, Nonlinear Anal. 13 (1989), 863869. MR 90g:54041

[So] P. Soardi, Existence of fixed points of nonexpansive mappings in certain Banach lattices, Proc. Amer. Math. Soc. 73 (1979), 25-29. MR 80c:47051

Department of Mathematical Sciences, The University of Texas at El Paso, El Paso, TEXAS 79968-0514

E-mail address: mohamed@math.utep.edu 